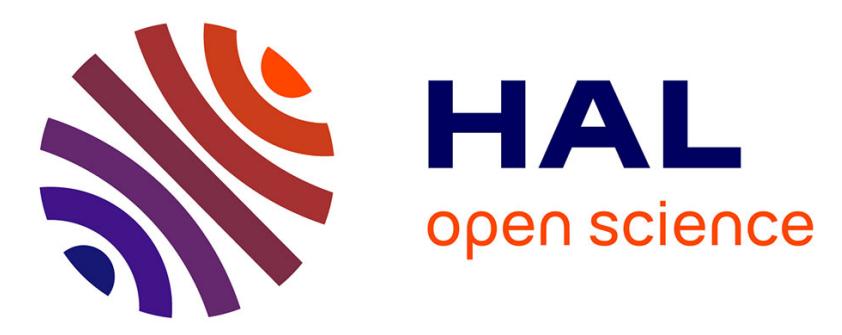

\title{
Game theoretical modelling of survival strategies of inside macrophages
}

\author{
S. Hummert, C. Hummert, A. Schröter, B. Hube, S. Schuster
}

\section{To cite this version:}

S. Hummert, C. Hummert, A. Schröter, B. Hube, S. Schuster. Game theoretical modelling of survival strategies of inside macrophages. Journal of Theoretical Biology, 2010, 264 (2), pp.312. 10.1016/j.jtbi.2010.01.022 . hal-00585791

\section{HAL Id: hal-00585791 https://hal.science/hal-00585791}

Submitted on 14 Apr 2011

HAL is a multi-disciplinary open access archive for the deposit and dissemination of scientific research documents, whether they are published or not. The documents may come from teaching and research institutions in France or abroad, or from public or private research centers.
L'archive ouverte pluridisciplinaire HAL, est destinée au dépôt et à la diffusion de documents scientifiques de niveau recherche, publiés ou non, émanant des établissements d'enseignement et de recherche français ou étrangers, des laboratoires publics ou privés. 


\section{Author's Accepted Manuscript}

Game theoretical modelling of survival strategies of Candida albicans inside macrophages

S. Hummert, C. Hummert, A. Schröter, B. Hube, S. Schuster

PII: S0022-5193(10)00034-2

DOI: doi:10.1016/j.jtbi.2010.01.022

Reference: $\quad$ YJTBI 5844

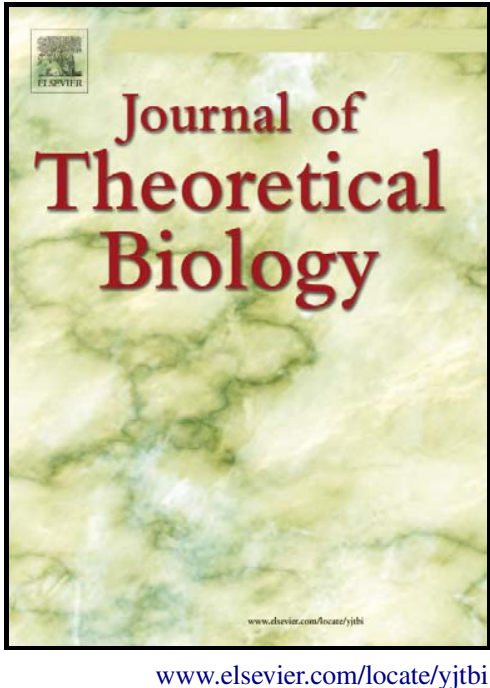

To appear in: $\quad$ Journal of Theoretical Biology

Received date: $\quad 23$ September 2009

Revised date: $\quad 17$ January 2010

Accepted date: 19 January 2010

Cite this article as: S. Hummert, C. Hummert, A. Schröter, B. Hube and S. Schuster, Game theoretical modelling of survival strategies of Candida albicans inside macrophages, Journal of Theoretical Biology, doi:10.1016/j.jtbi.2010.01.022

This is a PDF file of an unedited manuscript that has been accepted for publication. As a service to our customers we are providing this early version of the manuscript. The manuscript will undergo copyediting, typesetting, and review of the resulting galley proof before it is published in its final citable form. Please note that during the production process errors may be discovered which could affect the content, and all legal disclaimers that apply to the journal pertain. 


\title{
Game theoretical modelling of survival strategies of Candida albicans inside macrophages
}

\author{
S. Hummert*,a,b , C. Hummert ${ }^{\mathrm{b}}$, A. Schröter ${ }^{\mathrm{a}}$, B. Hube ${ }^{\mathrm{a}, \mathrm{b}}$, S. Schuster ${ }^{\mathrm{a}}$ \\ ${ }^{a}$ Friedrich-Schiller University, School of Biology and Pharmaceutics, Ernst-Abbe-Platz 2, \\ D-07743 Jena, Germany \\ ${ }^{b}$ Leibniz-Institute for Natural Product Research and Infection Biology - \\ Hans-Knöll-Institute, Beutenbergstr. 11a, D-07745 Jena, Germany
}

\begin{abstract}
The polymorphic fungus Candida albicans can live as an aggressive pathogen that causes a wide variety of diseases in humans. Host resistance against these infections is mediated predominantly by phagocytes, namely neutrophils and macrophages. This report provides two game theoretical models of ingested Candida albicans cells in macrophages. Two strategies are available for each pathogenic yeast cell: avoiding lysis transiently (called silencing) or forming hyphae and escaping (called piercing because the macrophage is pierced from inside). In dependence on parameter values, two different outcomes can be derived from the model: when the difference of the costs of the two strategies is low, all fungal cells inside a macrophage will play the piercing strategy, while in the high-cost case, a mixed population of piercing and silencing cells is the only stable solution. Further, the role of the $S A P$ gene family encoding secreted proteinases and the Sap proteins is investigated with the help of known studies and is put in relation to the costs of the strategies, the most important parameter of this model. Our results are in agreement with wet-lab results presented by other groups and the model parameters can be estimated from experimental data.
\end{abstract}

Key words: immune evasion, candidiasis, harmony game, hawk-dove game,

\footnotetext{
* Corresponding author

Email addresses: sabine.hummert@uni-jena.de (S. Hummert), christian.hummert@hki-jena.de (C. Hummert), an.schroeter@uni-jena.de (A. Schröter), bernhard.hube@hki-jena.de (B. Hube), stefan.schu@uni-jena.de (S. Schuster)
} 
playing the field

\section{Introduction}

The polymorphic fungus Candida albicans grows either in a unicellular yeast form or in elongated tubes known as hyphae and can switch growth modes between these stages. By branching, the hyphae can form large mycelia [9]. C. albicans can live both as a harmless commensal on the human skin and mucosal surfaces and as an aggressive pathogen that causes a wide variety of diseases (candidiasis), including oropharyngeal thrush and vaginitis. In patients with compromised immune functions, C. albicans can cause even life-threatening systemic infections at a broad range of body sites [26]. Due to the fact that the majority of the human population is carrier of this fungus, $C$. albicans can be considered as the most wide-spread opportunistic pathogenic fungus.

C. albicans has a high degree of flexibility and, thus, can exist and proliferate in environments that are extremely variable in oxygen and carbon dioxide levels, pH, osmolarity, availability of nutrients, and temperature [10, 20]. One of the most important virulence factors is the ability to undergo morphogenesis. Yeast cells are able to form germ tubes and then grow as hyphae.

Host resistance against infections with $C$. albicans is mediated predominantly by phagocytes, namely neutrophils and macrophages [12]. It has been shown that neutrophils are much more effective to kill the yeast pathogens than macrophages [22]. After phagocytosis, C. albicans is exposed to nitric oxide and reactive oxygen species, antimicrobial peptides, proteinases, extreme $\mathrm{pH}$ values and to a nutrient-poor environment [21, 14]. On the other hand, C. albicans possesses a variety of different strategies to escape the immune system and invade into deeper tissues. C. albicans is equipped with several genes and transcriptional programs that may help the fungus to counteract phagocytic attack. Fungal cells can avoid being lysed and killed by secreting proteins and by preventing the macrophages from combining the phagosome and lysosome to the phagolysosome [28, 2, 13]. Once inside a macrophage, the yeast form of $C$. albicans is able to differentiate into the hyphal form which can pierce and destroy the macrophage within a few hours after phagocytosis $[24,46]$. In contrast, $C$. albicans is not capable of piercing neutrophils [14]. In the filamentous morphology $C$. albicans itself lyses the macrophage from inside [25]. The resistance to lysis (defensive strategy, here 
called silencing) and the production of hyphal cells killing the macrophages from within (offensive strategy, here called piercing) are both important and independent possible escape strategies of the fungus.

Evolutionary game theory has turned out to be a powerful methodology for modelling the properties of organisms as resulting from evolution and coevolution $[17,18,34,35]$. Interacting organisms or species are considered as players that try to maximize their payoff (fitness). Game theory takes into account that the payoff for each player does not only depend on its own strategy but also on that of the other(s). This theory has been applied to the interplay (competition, cooperation etc.) both between different species and between different individuals or subpopulations of the same species. Examples of the former case are host-parasite interactions [37], cleaning symbiosis [36], or the origin of autumn colours as a result of coevolution between insects and trees [3]. For the latter case, examples are 'classical' animal conflict situations, such as two male individuals fighting for food, a territory or a potential mate [30], kleptoparasitism [8], or siblicide in the kittiwake gull [11]. Recently, game theory has also been used for studying the evolution of biochemical pathways [35], especially the use of two possible ATP producing pathways by two different strains of microorganisms [15]. Further applications concern tumour growth $[4,44,27]$ and investigations of the virus RNA phage $\Phi 6$ [45].

In this paper, two game theoretical models of the survival strategies of $C$. albicans yeast cells ingested by macrophages are presented. The players of the game are $C$. albicans yeast cells that are randomly chosen from the whole yeast cell population by ingestion by one macrophage. That macrophage provides the environmental condition in these models. The yeast cells inside the macrophage are assumed to choose between two different strategies. The silencing strategy enables the yeast cell transiently to survive intracellularly until other Candida cells that use the piercing strategy within the same macrophage kill the macrophage. Thus, the survival of fungal cells adopting the silencig strategy is dependent on the killing by others. A first simple model investigates the situation as a two player game by determining the Nash equilibria and the game type. A Nash equilibrium is a situation where none of the players would benefit from changing strategy unilaterally [18]. The second model is based on the more realistic assumption of a random number of yeast cells inside a macrophage. In that model, each cell is considered to play against several others simultaneously, which is, in game theory, called 'playing the field' [29] or 'population game' [18]. 


\section{Experimental Background}

There are different experimental studies comparing the virulence attributes of isolated C. albicans strains. Zeng et al. [47] found significant differences in adhesion to epithelial cells and in phospholipase activity between four different genotypes of $C$. albicans isolates from oral lichen planus patients. The relationship between genotype and enzymatic activity, especially extracellular proteinase and phospholipase activity, was investigated by Sugita et al. [39]. There, three genotypic strains were used. One strain had significantly higher proteinase and phospholipase activity compared with the other two genotypes. Similar examinations have been done by Nawrot et al. [33].

The group of Tavanti et al. [40] studied the pathogenic potential of $C$. albicans isolates with diverse genomic backgrounds, notably b and c karyotypes. The $C$. albicans isolates were assayed in cocultures with monocytic derived macrophages (THP-1 cells). C. albicans yeast cells and macrophages have been cocultured in the ratios 1:1 and 1:10. In the 1:1 case, the macrophages ingested $71.8 \%$ of the karyotype $\mathrm{b}$ and $51.8 \%$ of the karyotype c cells. This gives realistic values for the average number of ingested yeast cells by one macrophage between 0.5 and 1 . However, ingested cells are not equally distributed among the macrophages and often there are more than one ingested yeast cell inside one macrophage. In fact, the fraction of macrophages without any ingested yeast cell is probably large because if one macrophage did ingest a yeast cell, it becomes more active (as seen in [5]). The Tavanti work further showed that $\mathrm{b}$ and $\mathrm{c}$ karyotypes have a different pathogenic potential. Also, strains isolated from HIV patients showed an increased pathogenicity compared to isolates from healthy carriers. The recovery of viable fungal cells is expressed by the intracellular replication index (IRI), calculated from the ratio of recovered to ingested fungal cells. Note that fungal cells can proliferate inside phagocytes or they can be killed by them. By definition, an IRI ratio equaling 1 is obtained when the number of recovered $C$. albicans cells is equal to that of phagocytosed cells. Cells that were not ingested were not taken into account. The IRI of the c karyotype always exceeds 1 , ranging from 1.62 to 2.34. It was shown that all c karyotype isolates produce extensive hyphal forms inside macrophages. In contrast, the b karyotype showed IRIs ranging from 0.65 to 0.91 and about $69.3 \%$ of the cells were not able to undergo yeast-to-hyphae transition intracellularly.

Therefore, it may be an adopted strategy not to switch into the hyphal growth form and rather to survive as yeast cells only by using survival ac- 
tivities which do not kill the host cell. Considering the two growth forms as different survival strategies, it appears promising to model the interaction between them by game-theoretical methods.

C. albicans possesses a repertoire of secreted hydrolases, namely proteinases, lipases and phospholipases, which facilitate the pathogen invasion of the host cells without directly killing the host cell [19]. These enzymes may also allow the fungus to utilize host cell macromolecules as a source of nutrients. Proteinases are classified according to the catalytic type. Aspartic proteinases are predominantly active at acidic $\mathrm{pH}$ and play important roles in a range of diseases. The family of secreted aspartic proteinases (Sap) is possibly the best characterized set of proteins of $C$. albicans. Reviews providing extensive overviews about these proteinases are $[19,31]$. The $C$. albicans $S A P$ gene family consists of ten members. Expression of the $S A P$ genes depends on many factors including ambient $\mathrm{pH}$, temperature, and nutrient sources which vary according to the type and stage of the disease. SAP gene expression is also regulated during the morphological transition from yeast to hyphae, and during the phenotypic switch between white and opaque cells.

$S A P 1$ and $S A P 3$ are regulated by phenotypic switching and were found to be opaque-phase-specific. $S A P 2$ was expressed in both white and opaque phenotypes. SAP4-SAP6 were almost exclusively expressed during hyphal formation at near neutral $\mathrm{pH}$ values and were possibly required for invasion in vivo. Expression of $S A P 5$ and $S A P 6$ was also increased during the biofilm formation. $S A P^{7} 7$ was expressed during mucosal infection in mice [42]. SAP8 was temperature-regulated in vitro. SAP9 and SAP10 were shown to be expressed under several in vitro conditions and in patient samples [1, 32].

Sap isoenzymes were reported to be involved in adherence, colonization, nutrition and dissemination of $C$. albicans. Sap1-Sap3 were found to participate in adhesion of $C$. albicans to the host cells. Sap2 has very broad substrate specificity and can degrade many human proteins [31]. Thus, Sap2 could be important to supply the fungus with nutrients in nutrient-poor environments. Borg-von Zepelin et al. [7] investigated the expression of Saps from C. albicans within murine macrophages and observed Sap1 to Sap3 antigen inside and outside the macrophages on yeast cells and pseudohyphae. The detection of Sap1 to Sap3 was shown to be independent on the contact of fungal cells with macrophages. SAP4-6 gene products were required for systemic infections and were likely to contribute to virulence by helping $C$. albicans to resist the phagocytic attack [38]. Borg-von Zepelin et al. [7] demonstrated that Sap4 to Sap6 were produced in macrophages after 
phagocytosis of $C$. albicans and protect fungal cells from being destroyed. The presence of Sap4 to Sap6 was shown to be a consequence of the C. albicans-macrophage interaction, as no Sap4-6 proteinase antigens were detected on the surface of fungal cells outside the macrophages. Sap7 expression did not correlate with virulence in mice [42] and the role of Sap8 remains unknown. Sap9 and Sap10 were shown to play a role in cell wall integrity and in $C$. albicans adhesion to host cells [1]. In general, activities and functions of the Sap isoenzymes overlap. The role of the $S A P$ family in C. albicans pathogenesis is still under investigation.

Another study of Tavanti et al. [41] evaluated whether b and c karyotypes of $C$. albicans secrete different levels of Saps and express different patterns of the Sap-encoding genes SAP1-10. It was found that the mean value of Sap activity was significantly lower in the commensal than in the infectious b karyotype, whereas in the c type Sap activity was similar for both, the commensal and infectious strains. Commensal strains with the c karyotype shared the propensity to secrete higher levels of proteinases than strains with the b karyotype. Under non-Sap-inducing conditions none of the commensal b strains expressed $S A P$ 2, whereas all $S A P$ genes were expressed by commensal strains with the c karyotype. While all of the $S A P 1-10$ genes were detectable under Sap-inducing conditions, the timing of their expression during growth differed significantly in $\mathrm{c}$ and $\mathrm{b}$ commensal strains. The major finding of this study was the demonstration that $C$. albicans strains with $\mathrm{b}$ and $\mathrm{c}$ karyotypes are characterized by marked differences in the expression of SAP1-10 genes and by the ability to secrete significantly different levels of Saps during in vitro propagation. It appears that commensal C. albicans isolates with the c karyoype are more prone than the b strains to rapidly express all $S A P$ genes and to secrete higher Sap levels. This observation may suggest that $C$. albicans strains with the c karyotype may be more likely to behave as pathogens than the b type.

\section{Game theoretical modelling}

Assumptions and Strategies

We consider a large population of $C$. albicans yeast cells inside a human host meeting macrophages. Each macrophage ingests some yeast cells, this means a random sample of now interplaying fungal cells is taken.

The outcome (also called fitness) of an ingested yeast cell applying a certain strategy is denoted as $W\left(S_{1}, S_{2}\right)$, where the entry $S_{1}$ refers to the 
own adopted strategy and the $S_{2}$ to the counterpart's strategy. In the first model, the counterpart will be another yeast cell and in the second model it will be the whole population. The strategy of the whole population is here interpreted as an average strategy. The fitness can be measured in terms of the growth rate of $C$. albicans cells. Under certain environmental conditions, a 'standard' growth rate can be assumed.

The players in the game, i.e. the ingested yeast cells, can use one of two strategies: they may grow as hyphae and pierce the macrophage (piercing strategy - P), or they may silence by avoiding lysis transiently (silencing strategy - S).

Both the strategies $\mathrm{S}$ and $\mathrm{P}$ imply certain costs, leading to a decrease in growth rate. For $\mathrm{S}$ the production of certain substances is required whereas for the morphological switch $(\mathrm{P})$, a complex reconstruction of the actin skeleton and chromosomal rearrangement are additionally needed [23]. Therefore, it can be assumed that the costs involved in $\mathrm{P}$ are higher. The difference between the $\mathrm{P}$ and $\mathrm{S}$ costs is called $c$.

It is assumed that $\mathrm{P}$ is always successful and saves the live of the $C$. albicans cell adopting it. However, all other yeast cells that are inside this macrophage survive as well. Cells that stay alive (and payed the costs for S) get a payoff $l$.

The symmetric two player game

In a first simple model, it is assumed that out of a large yeast cell population exactly two yeast cells are ingested by one macrophage. This situation can be modelled by a symmetric two player game, where both $C$. albicans cells inside the macrophage play against each other and the macrophage provides the environment for the game. All other cells of the $C$. albicans population outside the macrophage as well as other macrophages do not play any role for this two player game. As mentioned before, the final situation in the game depends on the combination of the strategies. If both yeast cells inside the macrophage adopt $\mathrm{P}$, both will survive, each one paying the switching costs. In the case where both players decide to silence which involves lower costs, they end up by being killed by the macrophage. If the yeast cells play the respective other strategy, they both escape from the macrophage. However, the costs borne by the $\mathrm{P}$ strategist are higher, leading to an advantage in fitness for the $\mathrm{S}$ cell. The different cases of this game are illustrated in Figure 1 and the payoff matrix of this game is shown in Figure 2.

For $l<c$ the only Nash equilibrium is, when both the players choose $\mathrm{S}$, 
otherwise they would get a negative fitness. Such a game is called Harmony game [17]. If $c<l$, the two pure Nash equilibria lie on the secondary diagonal of the payoff matrix, that means this game is a hawk-dove game [18]. The name of this game comes from the situation where animals can opt either for an aggressive (hawk) or a peaceful (dove) strategy. The two Nash equilibria correspond to the situations where one player acts as a hawk and the other as a dove. As it might be somewhat counter-intuitive, it is worth noting that the hawk strategy here corresponds to the (non-cooperative) silencing strategy, while the dove strategy corresponds to piercing because the former gives a higher payoff provided that the partner plays the other strategy. The piercing strategy is peaceful (cooperative) with respect to the partner rather than with respect to the macrophage, which is the environment in this game.

The hawk strategy is aggressive against the partner, while the piercing strategy is peaceful, even cooperative against the partner. The aggressive behavior is against the macrophage which is environment in this game.

Applying this game to the whole population, it is useful to consider many two-player games, where the partners are chosen randomly from the population. Such a population consists of individuals with different strategies. A population that is stable against mutants is called evolutionary stable. The average population strategy (depending on the ratios of the different strategists) is called evolutionary stable strategy (ESS) [29]. The hawk-dove game has only one ESS, which is mixed. In a mixed ESS, different fractions of the population play different strategies. By using the Bishop-Cannings theorem [6], the ESS can be easily calculated as the frequency

$$
x=\frac{l-c}{l}
$$

of $\mathrm{P}$ strategists. According to this theorem, the ESS can be found when $W(P, x)=W(S, x)$, which occurs when $x W(P, P)+(1-x) W(P, S)=$ $x W(S, P)+(1-x) W(S, S)$. The same solution applies to the case where each cell switches strategies. The fraction $x$ can be interpreted as the average probability of a single cell playing $\mathrm{P}$.

The playing the field game

Using a more realistic assumption of an average number $\lambda>0$ of ingested yeast cells, it has been taken into account that one $\mathrm{P}$ strategist can help survive all other cells inside the macrophage. For this reason, the theory of playing the field [29] is applicable where the fitness of each single $C$. albicans cell in a pure or mixed population is considered. 
In a mixed population of $\mathrm{P}$ and $\mathrm{S}$ cells, let $x$ be the ratio of cells playing $\mathrm{P}$. Then $1-x$ is the ratio of cells playing $\mathrm{S}$. The special case $x=1$ represents the pure population of $\mathrm{P}$ cells and the case $x=0$ gives the pure population of $\mathrm{S}$ cells. Alternatively, $x$ can be interpreted as a mixed strategy of one cell by playing strategy $\mathrm{P}$ with probability $x$ and strategy $\mathrm{S}$ with probability $1-x$. A population consisting of $x$-strategists is equivalent to the previous mixed population.

Assuming macrophages trying to phagocytose yeast cells very often, and the probability of success being very low, the Poisson distribution is a good approximation for the underlying probability distribution. The survival probability of an ingested $\mathrm{S}$ cell is the probability that there is at least one P cell in the macrophage under the condition that there is at least one $\mathrm{S}$ cell in the macrophage. This survival probability is dependent on the ratio $x$. Because these events are stochastically independent this probability $p(x)$ can be calculated with the help of the contrary event that there is no $\mathrm{P}$ cell in the macrophage as

$$
p(x)=P(X \geq 1)=1-P(X=0)=1-e^{-\lambda x},
$$

where $X$ denotes the random variable that gives the number of ingested $\mathrm{P}$ cells.

In a pure population of $\mathrm{P}$ cells, all individuals survive, but for each one the costs arise, whereas a single cell that adopts $\mathrm{S}$ is saved 'for free' (only silencing costs apply) as long as there is at least one other $(\mathrm{P})$ cell inside the macrophage. That means this single $\mathrm{S}$ cell will survive with the probability $p(x=1)=1-e^{-\lambda}$. Note that in a large population one single $\mathrm{S}$ cell practically does not change $x$.

A population consisting only of $\mathrm{S}$ cells cannot survive $(p(x=0)=0)$, whereas a single $\mathrm{P}$ cell in this population would survive by paying the costs. See the fitness matrix in Figure 3 for reviewing all possible cases.

A pure population will now be evolutionary stable, if a single cell does not profit from playing the other strategy. This is never the case for the strategy $\mathrm{S}$, as long as $c<l$. A pure population of $C$. albicans cells that produce hyphae as soon as ingested by a macrophage, can be stable against $\mathrm{S}$ cells, if the condition $W(P, P)>W(S, P)$ holds, that means, the fitness for a single $\mathrm{P}$ cell is higher than for a single $\mathrm{S}$ cell.

In the case $W(P, P)<W(S, P)$, that means, if the condition

$$
\frac{l}{c}<e^{\lambda}
$$


holds, both pure populations are unstable and a mixed population can be calculated.

At equilibrium, the following condition must be fulfilled:

$$
W(P, x)=W(S, x) .
$$

To determine these fitnesses, the payoffs of the two cases (at least one $\mathrm{P}$ cell is present, no $\mathrm{P}$ cell is present beside the player) have to be added upon being weighted by the probabilities resulting from the Poisson distribution. For a $\mathrm{P}$ cell in both cases the payoff is $l-c$. For an $\mathrm{S}$ cell in the first case the payoff is $l$ and in the second case 0 .

$$
\begin{aligned}
W(P, x) & =W(S, x) \\
l-c & =p(x) l \\
l-c & =\left(1-e^{-\lambda x}\right) l \\
c & =e^{-\lambda x} \\
\bar{l} & =\frac{1}{\lambda} \ln \left(\frac{l}{c}\right) .
\end{aligned}
$$

In order that this value is indeed a ratio, it has to be in the intervall $(0,1)$. This is guaranteed due to conditions $c<l$ and (3).

To prove the evolutionary stability of this state, a small part $\varepsilon>0$ in the population playing another strategy $y \neq x$ is considered, so that the average population plays now the strategy

$$
z=\varepsilon y+(1-\varepsilon) x=\varepsilon(y-x)+x .
$$

Cells playing S now survive with probability $p(z)$ (equation (2)). Evolutionary stability for the strategy $x$ is given, if

$$
\begin{aligned}
W(y, z) & <W(x, z) \\
y(l-c)+(1-y) p(z) l & <x(l-c)+(1-x) p(z) l \\
y(l-c-p(z) l) & <x(l-c-p(z) l),
\end{aligned}
$$

meaning that the new strategy $y$ in the new population $z$ cannot do as well as the equilibrium strategy $x$ and $x$ will establish again. In fact, this can be proven for the calculated $x$ by distinguishing the two cases $x<y$ and $x>y$, leading to $p(z)>p(x)$ and $p(z)<p(x)$, respectively and regarding the 
equilibrium condition $l-c=p(x) l$. Figure 4 shows the different evolutionary stable populations in dependence on the parameter values.

The experimental investigations of the Tavanti group [40] include the observation of intracellular killing of $C$. albicans and killing rates are determined. For the presented game theoretical model, equivalent rates can be calculated. We only consider yeast cells that are ingested at some time point. For these, the survival probability of a single cell in the mixed population, also interpretable as survival rate of the whole population can be determined as follows. Let $U$ denote the event to survive. Then $P(U)$ can be calculated as follows:

$$
P(U)=x+(1-x)\left(1-e^{-\lambda x}\right)
$$

inserting $x$ (equation (5)):

$$
P(U)=1-\frac{c}{l}+\left(\frac{1}{\lambda} \ln \left(\frac{l}{c}\right)\right) \frac{c}{l} .
$$

This function is shown in Figure 5. For values $l / c<1$ it has to be set to zero, because in this case no yeast cell can afford the costs for the piercing strategy. With the critical threshold $c=l$ the probability becomes positive and increases with increasing ratio $l / c$ until $l / c=\exp (\lambda)$ (condition (3)), where it reaches unity. For values $l / c>\exp (\lambda)$ there are no $\mathrm{S}$ strategists but a pure $\mathrm{P}$ population and $P(U)$ has to be set to one.

\section{Discussion}

In this work, we present a game-theoretical model of ingested Candida albicans cells in macrophages. Two strategies are available for each cell: secreting proteins and avoid lysis (called silencing) and additionally forming hyphae and escape (called piercing here because the macrophage is pierced from inside). The macrophages are assumed as a constant environment, so only $C$. albicans cells are considered as players. From the model, we derived two different outcomes: when the difference of the costs of the two strategies is small, all fungal cells inside a macrophage will play the piercing strategy, while in the high-cost case, a mixed population of piercing and silencing cells is the only stable solution. This is in good agreement with the experimental observation of the two karyotypes studied by Tavanti et al. [41]. 
A coexistence of two subpopulations using different strategies is frequently also observed in other species including higher animals. For example, a normal breeding strategy and a 'duck and cover' strategy (comparable to the silencing strategy) have been observed in voles in the presence of predators [16], and different strains of the bacteriophage $\Phi 6$ can coexist, with defectors containing less genetic material for replication enzymes [45].

As a special case, we first analysed the two-player game, that is, the situation where exactly two cells are ingested. This consideration leads to a hawk-dove game with the two Nash equilibria where the two players adopt different strategies. Independently from the value of the benefit-to-cost ratio $(l / c>1)$, this game has a mixed evolutionary stable population. The ratio of piercing yeast cells decreases with increasing costs. In contrast, the playing the field model also allows a pure piercing population if we use an average number of ingested cells of two. The difference between these two model outcomes is due to the different assumption of the exact (two-player game) or average (playing the field model) number of two ingested cells. We presented the two player game for didactic reasons to better explain the game-theoretical modelling.

As for the parameters in the playing the field model, we can see that for a larger average number of ingested yeast cells $\lambda$ the costs $c$ have to be very low in relation to the payoff $l$ for the pure piercing population to be evolutionarily stable. From the ratio $x$ (equation (5)), it can be seen that the costs for hyphae formation can be estimated from the experimentally observed fraction of cells showing hyphal growth. The estimate is made by using the calculated monotonic decreasing dependence between these two quantities.

The number of yeast cells ingested by one macrophage is strongly dependent on environmental conditions. A broad range of ingestion numbers is conceivable under certain conditions in vivo. As mentioned in section 2, observed ingestion numbers are between 0.5 and 1 . The video of a $C$. albicans - macrophages coculture in the work of Behnsen et al. [5], however, shows seven yeast cells ingested by one macrophage. Assuming the value $\lambda=7$, the $l / c$ ratio equals 1100 at the boundary line between the two populations (Figure 5). Assuming another macrophage to yeast cells ratio, maybe an average number of 7 ingested cells becomes realistic also in the work of Tavanti et al. [40]. Then the following theoretical consideration can be made. Since not all ingested cells of the b karyotype survived, for this genotype $l / c<1100$ should hold, whereas the c karyotype (nearly all blastospores grew as hyphae 
inside macrophages) fulfills $l / c>1100$. Considering an average number of 0.7 ingested cells (Tavanti work [40], 1:1 ratio of macrophages and yeast cells), the $l / c$ ratio equals 2.23 at the boundary line between the mixed and the pure piercing population. Knowing the IRI $(0.65<$ IRI $<0.91)$ for the b karyotype and comparing it to the survival rate $P(U)$ of the model, $l / c$ can be determined more exactly as $1.4<l / c<1.8$. The model does not take proliferation into account, so IRIs larger than one (c karyotype) are not comparable with the survival rate. It seems that the cells of the $\mathrm{c}$ karyotype build a pure piercing population and the cells of the b karyotype are an evolutionary stable mixed population.

If fitness is measured in growth rates, it may be possible to quantify the colony forming units (cfus) (the number of living cells which can establish a colony) of cells of the c karyotype. The number of cfus could be compared between cells grown under normal growth conditions versus cells co-incubated with macrophages. The differences in cfus may give an estimate for the costs for piercing plus silencing. However, the costs c represent the cost for piercing without silencing. The costs of silencing as a strategy may be estimated by comparing the cfus of mutant cells which cannot pierce macrophages versus wild type cells which can. However, these mutant cells often lack more biological activites in addition to the ability to pierce and escape. Similar experiments are planned for future studies.

The model states that a non-piercing strain would benefit from the presence of a piercing strain. In terms of experiments, the IRI for a b karyotype strain would be expected to increase if the c karyotype is added for a short time. With increasing fraction $x$ of piercing cells, the model predicts an increasing fitness $W(S, x)$ and an increasing survival probability $P(U)$ which can be compared to the IRI. In the long term, an addition of c karyotype cells can be considered as a small perturbation of the equilibrium. The evolutionary stability would then guarantee a reduction of piercing cells of the b karyotype.

As described in the experimental background, it was reported that $C$. albicans isolates with c karyotype express higher levels of $S A P$ genes and are able to secrete higher levels of Saps (proteins) than strains with b karyotype during their in vitro propagation [41]. Especially the $S A P 2$ gene was reported to be more frequently expressed by $\mathrm{c}$ than by b karyotypes. It is highly probable that the main role of the $C$. albicans Sap2 is to provide nutrition for the fungal cell [31]. The proteinases Sap4-6 are co-regulated during hyphal formation and support penetration and evading the immune response. In this 
regard, the costs $c$ can be interpreted as relative costs. For cells having large amounts of nutrients available, the high costs of the morphological switch do not play such an important role as for cells having only limited nutrient supply. Since the Sap2 proteinases provide cell nutrition, relative costs for the yeast-to-hyphae transition may be lower for Sap2 producing cells. In fact, the c karyotype expressing a higher number of $S A P$ genes always tends to follow the piercing strategy whereas the b karyotype expressing lower numbers of $S A P$ genes form a mixed population with some cells relying on silencing, possibly because they cannot afford the morphological switch. Also the survival rate is lower for the b karyotype. This model would support that the nutrients play a major role in $C$. albicans pathogenicity. Due to these observations, it can be hypothesized that the main difference between the two karyotypes is the expression level of $S A P 2$. If the karyotype b had more nutritions available, it would possibly produce also more Sap4-6 and form hyphae.

As the total gene content seems to be very stable in the genomes of different $C$. albicans strains, Thewes et al. [43] concluded that differences in virulence between these strains are likely due to changes in the expression levels of certain genes. This conclusion further supports the hypothesized relation between costs (gene expression and protein secretion) and virulence (immune evasion) above.

The present modelling study shows that game theory is a useful tool to describe the behaviour of polymorphic fungi in quantitative terms. The results meet with the wet-lab results from the literature and the model parameters can be estimated from experimental data.

\section{Acknowledgements}

The work was supported by the Leibniz Association. The authors are grateful to Dr. Bernd Krause for many helpful discussions.

\section{References}

[1] Albrecht, A., Felk, A., Pichova, I., Naglik, J. R., Schaller, M., de Groot, P., MacCallum, D., Odds, F. C., Schäfer, W., Klis, F., Monod, M., Hube, B., January 2006. Glycosylphosphatidylinositol-anchored proteases of Candida albicans target proteins necessary for both cellular processes and host-pathogen interactions. Journal of Biological Chemistry 281 (2), 688-694. 
[2] Alvarez-Peral, F. J., Zaragoza, O., Pedreno, Y., Argüelles, J.-C., August 2002. Protective role of trehalose during severe oxidative stress caused by hydrogen peroxide and the adaptive oxidative stress response in Candida albicans. Microbiology 148 (8), 2599-2606.

[3] Archetti, M., August 2000. The origin of autumn colours by coevolution. Journal of Theoretical Biology 205 (4), 625-630.

[4] Basanta, D., Hatzikirou, H., Deutsch, A., June 2008. Studying the emergence of invasiveness in tumours using game theory. The European Physical Journal B 63 (8), 393-397.

[5] Behnsen, J., Narang, P., Hasenberg, M., Gunzer, F., Bilitewski, U., Klippel, N., Rohde, M., Brock, M., Brakhage, A. A., Gunzer, M., February 2007. Environmental dimensionality controls the interaction of phagocytes with the pathogenic fungi Aspergillus fumigatus and Candida albicans. PLoS Pathogens 3 (2), e13.

[6] Bishop, D. T., Cannings, C., January 1978. A generalized war of attrition. Journal of Theoretical Biology 70 (1), 85-124.

[7] Borg-von Zepelin, M., Beggah, S., Boggian, K., Sanglard, D., Monod, M., May 1998. The expression of the secreted aspartyl proteinases sap4 to sap6 from Candida albicans in murine macrophages. Molecular Microbiology 28 (3), 543-554.

[8] Broom, M., Luther, R. M., Ruxton, G. D., Rychtář, J., August 2008. A game-theoretic model of kleptoparasitic behavior in polymorphic populations. Journal of Theoretical Biology 255 (1), 81-91.

[9] Calderone, R. A., 2002. Candida and Candidiasis. Blackwell Scientific Publications, Oxford.

[10] Calderone, R. A., Fonzi, W. A., July 2001. Virulence factors of Candida albicans. Trends in Microbiology 9 (7), 327-355.

[11] Dickins, D. W., Clark, R. A., April 1987. Games theory and siblicide in the kittiwake gull, Rissa tridactyla. Journal of Theoretical Biology 125 (3), 301-305. 
[12] Edwards, J. E. J., Rotrosen, D., Fontaine, J. W., Haudenschild, C. C., Diamond, R. D., May 1987. Neutrophil-mediated protection of cultured human vascular endothelial cells from damage by growing Candida albicans hyphae. Blood 69 (5), 1450-1457.

[13] Fernandez-Arenas, E., Bleck, C. K. E., Nombela, C., Gil, C., Griffiths, G., Diez-Orejas, R., April 2009. Candida albicans actively modulates intracellular membrane trafficking in mouse macrophage phagosomes. Cellular Microbiology 11 (4), 560-589.

[14] Fradin, C., De Groot, P., MacCallum, D., Schaller, M., Klis, F., Odds, F. C., Hube, B., April 2005. Granulocytes govern the transcriptional response, morphology and proliferation of Candida albicans in human blood. Molecular Microbiology 56 (2), 397-415.

[15] Frick, T., Schuster, S., June 2003. An example of the prisoner's dilemma in biochemistry. Naturwissenschaften 90 (7), 327-331.

[16] Fuelling, O., Halle, S., Jan 2004. Breeding suppression in free-ranging grey-sided voles under the influence of predator odour. Oecologia 138 (1), 151-159.

[17] Hauert, C., 2002. Effects of space in 2 x 2 games. International Journal of Bifurcation and Chaos 12 (7), 1531-1548.

[18] Hofbauer, J., Sigmund, K., 1998. Evolutionary Games and Replicator Dynamics. Cambridge University Press, Cambridge.

[19] Hruskova-Heidingsfeldova, O., May 2008. Secreted proteins of Candida albicans. Frontiers in Bioscience 1 (13), 7227-7242.

[20] Hube, B., August 2004. From commensal to pathogen: stage- and tissuespecific gene expression of Candida albicans. Current Opinion in Microbiology 7 (4), 336-341.

[21] Hube, B., August 2006. Infection-associated genes of Candida albicans. Future Microbiology 1 (2), 209-218.

[22] Lehrer, R. I., Cline, M. J., June 1969. Interaction of Candida albicans with human leukocytes and serum. Journal of Bacteriology 98 (3), 9961004 . 
[23] Loeb, J. D. J., Sepulveda-Becerra, M., Hazan, I., Liu, H., June 1999. A $\mathrm{g}_{1}$ cyclin is necessary for maintenance of filamentous growth in Candida albicans. Molecular and Cellular Biology 19 (6), 4019-4027.

[24] Lorenz, M. C., Bender, J. A., Fink, G. R., October 2004. Transcriptional response of Candida albicans upon internalization by macrophages. Eukaryotic Cell 3 (5), 1076-1087.

[25] Lorenz, M. C., Fink, G. R., October 2002. Life and death in a macrophage: role of the glyoxylate cycle in virulence. Eukaryotic Cell 1 (5), 657-662.

[26] Macphail, G. L., Taylor, G. D., Buchanan-Chell, M., Ross, C., Wilson, S., Kureishi, A., June 2002. Epidemiology, treatment and outcome of candidemia: a five-year review at three canadian hospitals. Mycoses 45 (5-6), 141-145.

[27] Mansury, Y., Diggory, M., Deisboeck, T. S., January 2006. Evolutionary game theory in an agent-based brain tumor model: Exploring the 'genotype-phenotype' link. Journal of Theoretical Biology 238 (1), 146156.

[28] Martínez-Esparza, M., Martínez-Vicente, E., González-Párraga, P., Ros, J. M., Garcia-Peñarrubia, P., Argüelles, J.-C., February 2009. Role of trehalose-6p phosphatase (tps2) in stress tolerance and resistance to macrophage killing in Candida albicans. Journal of Medical Microbiology 19.

[29] Maynard Smith, J., 1982. Evolution and the Theory of Games. Cambridge University Press, Cambridge.

[30] Maynard-Smith, J., Price, G. R., November 1973. The logic of animal conflict. Nature 246 (5427), 15-18.

[31] Naglik, J. R., Challacombe, S. J., Hube, B., September 2003. Candida albicans secreted aspartyl proteinases in virulence and pathogenesis. Microbiology and Molecular Biology Reviews 67 (3), 400-428.

[32] Naglik, J. R., Moyes, D., Makwana, J., Kanzaria, P., Tsichlaki, E., Weindl, G., Tappuni, A. R., Rodgers, C. A., Woodman, A. J., Challacombe, S. J., Schaller, M., Hube, B., November 2008. Quantitative 
expression of the Candida albicans secreted aspartyl proteinase gene family in human oral and vaginal candidiasis. Microbiology 154, 32663280 .

[33] Nawrot, U., Skala, J., Wlodarczyk, K., Fonteyne, P., Nolard, N., Nowicka, J., 2008. Proteolytic activity of clinical Candida albicans isolates in relation to genotype and strain source. Polish Journal of Microbiology 57 (1), 27-33.

[34] Perc, M., Szolnoki, A., October 2009. Coevolutionary games - a mini review. Biosystems.

[35] Pfeiffer, T., Schuster, S., January 2005. Game-theoretical approaches to studying the evolution of biochemical systems. Trends in Biochemical Sciences 30 (1), 20-25.

[36] Poulin, R., Vickery, W. L., July 1995. Cleaning symbiosis as an evolutionary game: To cheat or not to cheat? Journal of Theoretical Biology 175 (1), 63-70.

[37] Renaud, F., de Meeüs, T., October 1991. A simple model of host-parasite evolutionary relationships. parasitism: Compromise or conflict? Journal of Theoretical Biology 152 (3), 319-327.

[38] Sanglard, D., Hube, B., Monod, M., Odds, F. C., Gow, N. A., September 1997. A triple deletion of the secreted aspartyl proteinase genes sap4, sap5, and sap6 of candida albicans causes attenuated virulence. Infection and Immunity 65 (9), 3539-3546.

[39] Sugita, T., Kurosaka, S., Yajitate, M., Sato, H., Nishikawa, A., 2002. Extracellular proteinase and phospholipase activity of three genotypic strains of a human pathogenic yeast, Candida albicans. Microbiology and Immunology 46 (12), 881-883.

[40] Tavanti, A., Campa, D., Bertozzi, A., Pardini, G., Naglik, J. R., Barale, R., Senesi, S., March 2006. Candida albicans isolates with different genomic backgrounds display a differential response to macrophage infection. Microbes and Infection 8 (3), 791-800.

[41] Tavanti, A., Pardini, G., Campa, D., Davini, P., Lupetti, A., Senesi, S., October 2004. Differential expression of secretory aspartyl proteinase 
genes (SAP1-10) in oral Candida albicans isolates with distinct karyotypes. Journal of Clinical Microbiology 42 (10), 4726-4734.

[42] Taylor, B. N., Hannemann, H., Sehnal, M., Biesemeier, A., Schweizer, A., Röllinghoff, M., Schröppel, K., October 2005. Induction of sap7 correlates with virulence in an intravenous infection model of candidiasis but not in a vaginal infection model in mice. Infection and Immunity 73 (10), 7061-7063.

[43] Thewes, S., Moran, G. P., Magee, B. B., Schaller, M., Sullivan, D. J., Hube, B., October 2008. Phenotypic screening, transcriptional profiling, and comparative genomic analysis of an invasive and non-invasive strain of Candida albicans. BMC Microbiology 8, 187.

[44] Tomlinson, I. P. M., August 1997. Game-theory models of interactions between tumour cells. European Journal of Cancer 33 (9), 1495-1500.

[45] Turner, P., Chao, L., March 2003. Escape from prisoner's dilemma in rna phage phi6. The American Naturalist 161 (3), 497-505.

[46] Vázquez-Torres, A., Balish, E., June 1997. Macrophages in resistance to candidiasis. Microbiology and Molecular Biology Reviews 61 (2), 170192.

[47] Zeng, X., Xiong, C., Wang, Z., Jiang, L., Hou, X., Shen, J., Zhou, M., Chen, Q., April 2008. Genotypic profiles and virulence attributes of Candida albicans isolates from patients with oral lichen planus. APMIS 116 (4), 284-291. 


\section{Figure Captions}

\section{Figure 1: Yeast macrophage interaction}

Hyphal growth and intracellular survival in a macrophage as two escape strategies of $C$. albicans from macrophage attack: a) a macrophage in a yeast cell population before ingestion, b) the two ingested yeast cells silence, c) the two ingested yeast cells pierce, d) the two ingested yeast cells adopt different strategies

Figure 2: Payoff matrix of the symmetric two player model In each field, the fitness of a yeast cell 'Cell 1' playing against a 'Cell 2' is given.

Figure 3: Fitness matrix of the playing the field game.

In each field, the fitness of a single yeast cell (row) playing against a pure population (column) is given.

\section{Figure 4: Evolutionary stable populations}

Evolutionary stable populations in dependence on the parameter sets: exponential relationship between $l / c$ and $\lambda$ according to condition (3).

\section{Figure 5: Survival rates}

Survival rates $P(U)$ of phagocytosed yeast cells in dependence on the benefitto-cost ratio. Parameter value: $\lambda=7$. 


\begin{tabular}{|c|c|c|c|}
\hline & \multicolumn{2}{|c|}{ Cell 2} \\
\hline & & piercing & silencing \\
\hline \multirow{2}{*}{ Cell 1} & piercing & $W(P, P)=I-C$ & $W(P, S)=I-C$ \\
\hline & silencing & $W(S, P)=1$ & $W(S, S)=0$ \\
\hline
\end{tabular}

\section{piercing silencing}

$W(P, P)=I-C \quad W(P, S)=I-C$

$$
W(S, P)=1 \quad W(S, S)=0
$$




\begin{tabular}{|cc|cc|}
\hline & & piercing & sopulation \\
& & $\mathrm{W}$ & \\
& piercing & $\mathrm{W}, \mathrm{P})=\mathrm{I}-\mathrm{c}$ & $\mathrm{W}(\mathrm{P}, \mathrm{S})=\mathrm{I}-\mathrm{C}$ \\
Individual & & & \\
& silencing & $\mathrm{W}(\mathrm{S}, \mathrm{P})=1-\mathrm{e}^{-\lambda}$ & $\mathrm{W}(\mathrm{S}, \mathrm{S})=0$ \\
\hline
\end{tabular}

piercing

silencing
$W(S, P)=1-e^{-\lambda}$ 


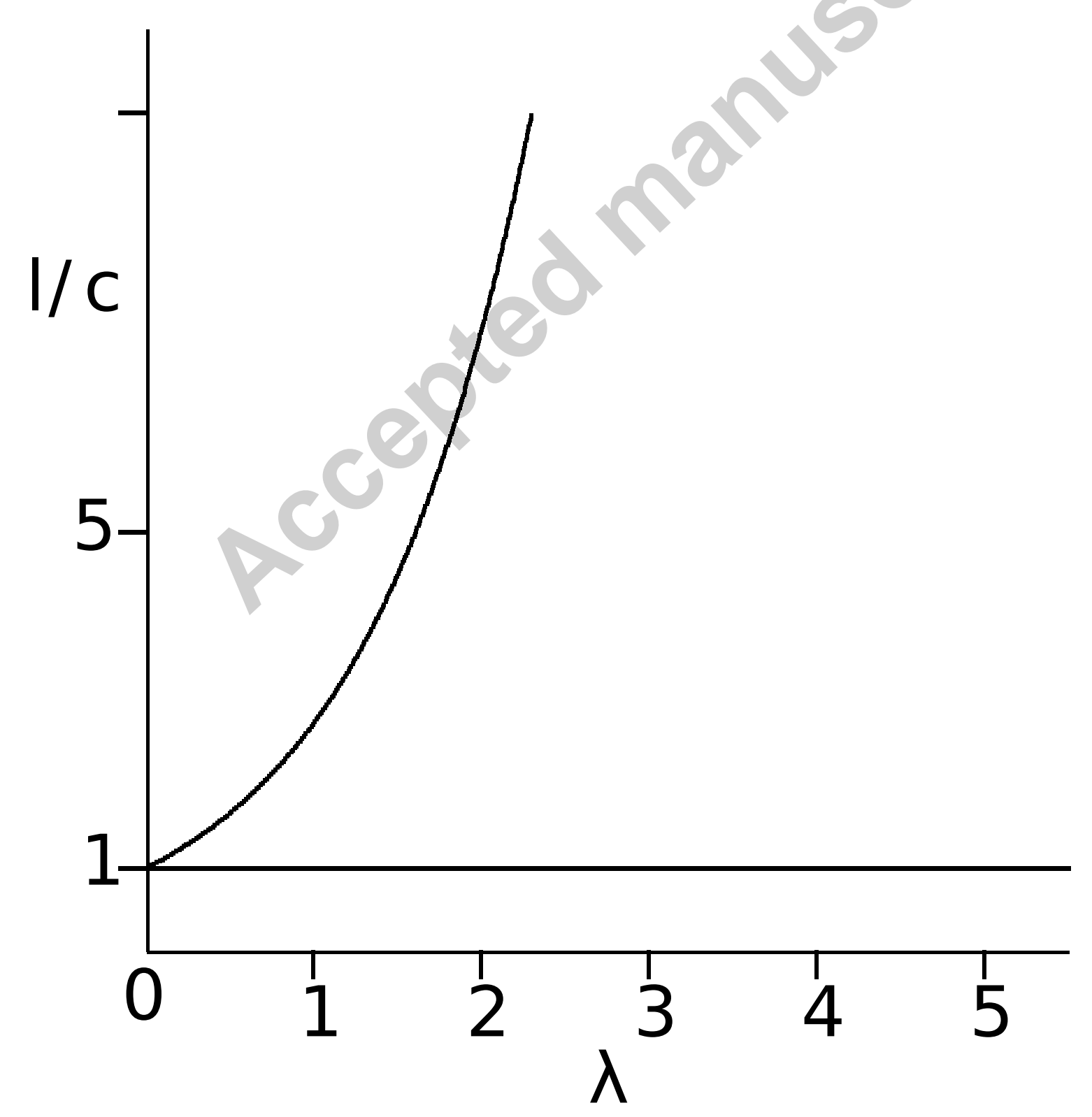

Figure 4 


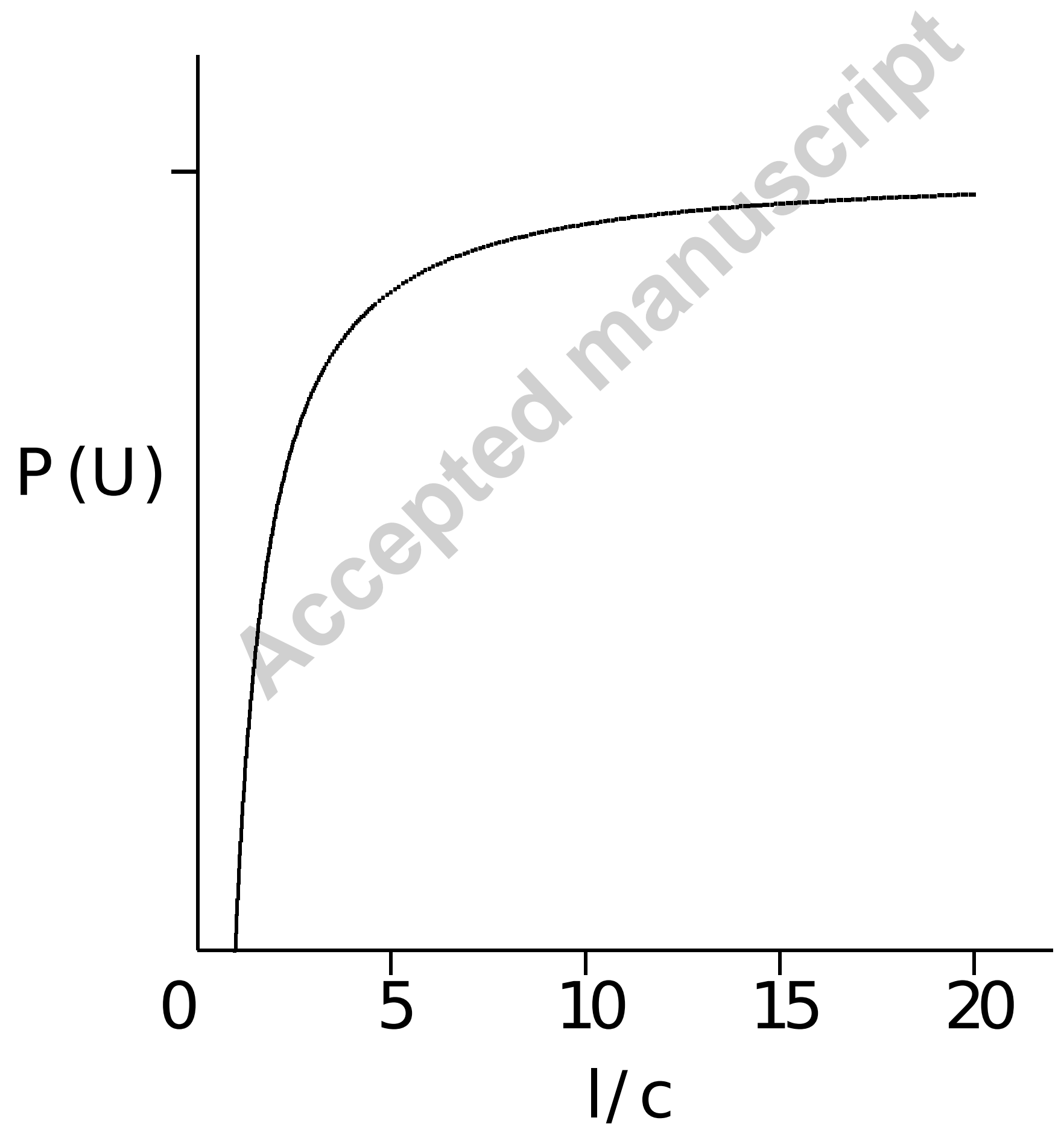

Figure 5 